\title{
The calcium-sensing receptor and vitamin $D$ receptor expression in tertiary hyperparathyroidism
}

\author{
TOMASZ GRZELA ${ }^{1}$, WITOLD CHUDZINSKI ${ }^{2}$, ZOFIA LASIECKA $^{4}$, JUSTYNA NIDERLA $^{1}$, \\ GRZEGORZ WILCZYNSKI ${ }^{4}$, BARBARA GORNICKA ${ }^{4}$, ALEKSANDER WASIUTYNSKI ${ }^{4}$, MAGDALENA DURLIK ${ }^{3}$, \\ ANNA BOSZCZYK ${ }^{1}$, ROBERT BRAWURA-BISKUPSKI-SAMAHA ${ }^{1}$, PIOTR DZIUNYCZ ${ }^{1}$, LUKASZ MILEWSKI ${ }^{1}$, \\ MARTA LAZARCZYK ${ }^{1}$, MACIEJ LAZARCZYK ${ }^{1}$ and IRENEUSZ NAWROT ${ }^{2}$ \\ ${ }^{1}$ Department of Histology and Embryology, Biostructure Research Center; ${ }^{2}$ Department of General, Vascular and Transplant \\ Surgery, Central University Hospital; ${ }^{3}$ Department of Clinical Immunology, Institute of Transplantology; \\ ${ }^{4}$ Department of Pathology, Warsaw Medical University, Warsaw, Poland
}

Received November 21, 2005; Accepted December 28, 2005

\begin{abstract}
The parathormone (PTH) production is controlled by calcium and vitamin $\mathrm{D}$, which interact with the calciumsensing receptor $(\mathrm{CaSR})$ and vitamin $\mathrm{D}$ receptor (VDR), respectively. All of these elements control calcium homeostasis, which is crucial for many physiological processes. Thus, impairment of the upstream component of this system, e.g. a decrease of CaSR and/or VDR, could result in hyperparathyroidism (HPTH). Therefore, the aim of this study was to assess the expression of CaSR and VDR in a tertiary form of HPTH (T-HPTH). The study involved 19 T-HPTH patients qualified for parathyroidectomy and 21 control parathyroids harvested from multi-organ cadaver donors. The small fragments of harvested glands were homogenized and used for Western blot analysis, whereas the remaining tissues underwent routine hematoxylin-eosin staining or immunostaining for CaSR and VDR. Among 64 T-HPTH parathyroids, 58 revealed the morphology of benign hyperplasia, 2 were identified as adenoma and 4 were classified as normal; some glands displayed a mixed histological phenotype. Western blot analysis revealed a decrease of CaSR and VDR in hyperplasia and adenomaderived samples. However, no correlation between the types of hyperplasia and receptor expression was observed. On the other hand, microscopic analysis of CaSR- and VDRimmunostained sections revealed a highly differentiated and
\end{abstract}

Correspondence to: Dr Tomasz Grzela, Cell Molecular Biology Laboratory, Department of Histology and Embryology, Biostructure Research Center, Warsaw Medical University, Chalubinskiego 5, PL 02004 Warsaw, Poland

E-mail: tgrzela@op.pl tgrzela@ib.amwaw.edu.pl

Key words: calcium-sensing receptor, parathyroid gland, tertiary hyperparathyroidism, vitamin D receptor significantly decreased mean expression of both receptors, which correlated with parathyroid histology. The reason behind the impaired expression of CaSR and VDR in T-HPTH is unclear. It presumably results from constant parathyroid stimulation at the stage of S-HPTH, followed by further development of polyclonal autonomy. However, the verification of this thesis requires further study.

\section{Introduction}

The production and secretion of parathormone (PTH), a main hormone produced by chief cells of parathyroid glands, remains under the control of calcium ions and vitamin $\mathrm{D}$ metabolites. The target molecules for these two key regulatory factors are calcium-sensing receptor (CaSR) and vitamin D receptor (VDR), respectively (1).

Calcium-sensing receptor, which belongs to the class III of G-protein coupled receptors, could be activated by various di- and tri-valent cations, especially $\mathrm{Ca}^{2+}$ and $\mathrm{Mg}^{2+}$, but also L-amino acids and other positively charged molecules (2). The triggering of CaSR by these agonists has been shown to post-transcriptionally down-regulate PTH expression, primarily by influencing the PTH-mRNA stability (1). The activation of CaSR also appears to inhibit parathyroid cell proliferation, however, the detailed molecular mechanism of this action remains unclear (3). Presumably, a useful model for the more precise determination of CaSR biological significance could be patients with loss-of-function receptor mutations, resulting in familial benign (hypocalciuric) hypercalcaemia $(\mathrm{FBHH})$ or neonatal severe primary hyperparathyroidism (NSP-HPTH) (4).

The receptor for vitamin D is the intracellular phosphoprotein belonging to the nuclear receptor superfamily (5). The triggering of VDR by its specific ligand results in its serine residue hyperphosphorylation and the formation of homodimeric or, in association with the 9-cis retinoic acid receptor (RXR), heterodimeric complexes. In the presence of co-activators, they interact directly with the DNA-based vitamin D response element (VDRE) and regulate PTH gene transcription (6). 


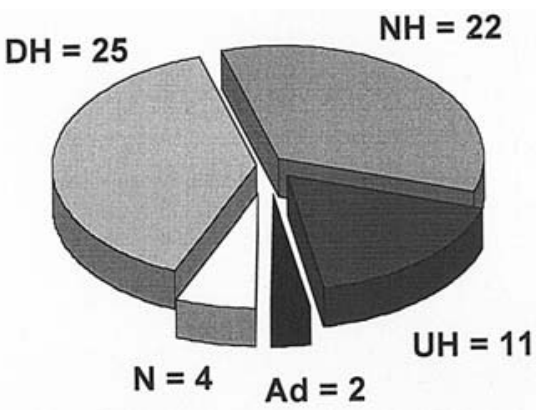

Figure 1. The distribution of the histological types of parathyroid glands derived from T-HPTH patients. $\mathrm{N}$, normal; $\mathrm{DH}$, diffused hyperplasia; $\mathrm{NH}$, nodular hyperplasia; UH, unclassifed/mixed hyperplasia; Ad, adenoma.

The chronic kidney insufficiency, due to a decrease of active vitamin D metabolites, may lead to disturbances in PTH, calcium regulation and the development of secondary hyperparathyroidism (S-HPTH) (7). This condition is often transient and, after the elimination of kidney insufficiency, e.g. successful kidney transplantation, results in the restoration of calcium balance. In some patients, however, even a kidney transplantation does not improve the course of S-HPTH; this is known as tertiary HPTH (T-HPTH) (8). The reason for the development of T-HPTH remains unclear. Some authors suggest that the long-term stimulation of parathyroids in SHPTH results in their hyperplasia. Presumably, it could also induce benign transformation and overt autonomy of glands in T-HPTH as proposed by some investigators (9). A similar condition predominantly characterizes primary HPTH (PHPTH) with the adenoma morphology of parathyroids and high levels of serum PTH.

PTH is the main factor responsible for increasing calcium concentration, which in turn, inhibits hormone production. This negative feedback regulation together with calcitonin, the hormone antagonizing the PTH action, enables maintenance of the plasma ionized calcium level within a very narrow range. Such tight control is necessary due to the crucial role of $\mathrm{Ca}^{2+}$ in many physiological processes, including bone mineralization, muscle contraction, neuronal excitability, and blood coagulation (10). Therefore, the impairment of upstream elements involved in calcium homeostasis, CaSR and/or VDR, could result in severe clinical complications, including death.

It has been proven that the expression of both CaSR and VDR molecules is down-regulated in patients with P-HPTH and S-HPTH (11-13). However, the role of CaSR and VDR in the pathogenesis of T-HPTH remains to be elucidated. Therefore, the aim of this study was to investigate the parathyroid expression of CaSR and VDR and their presumed correlation with gland microscopic morphology in T-HPTH individuals.

\section{Patients and methods}

Patients. Nineteen patients, 9 women and 10 men, who qualified for surgical treatment of T-HPTH were involved in the study. The mean age of patients was $43 \pm 10.4$ years (median, 46 years), and the mean duration of T-HPTH (after kidney transplantation) was $34 \pm 26.4$ months (median, 33 months).
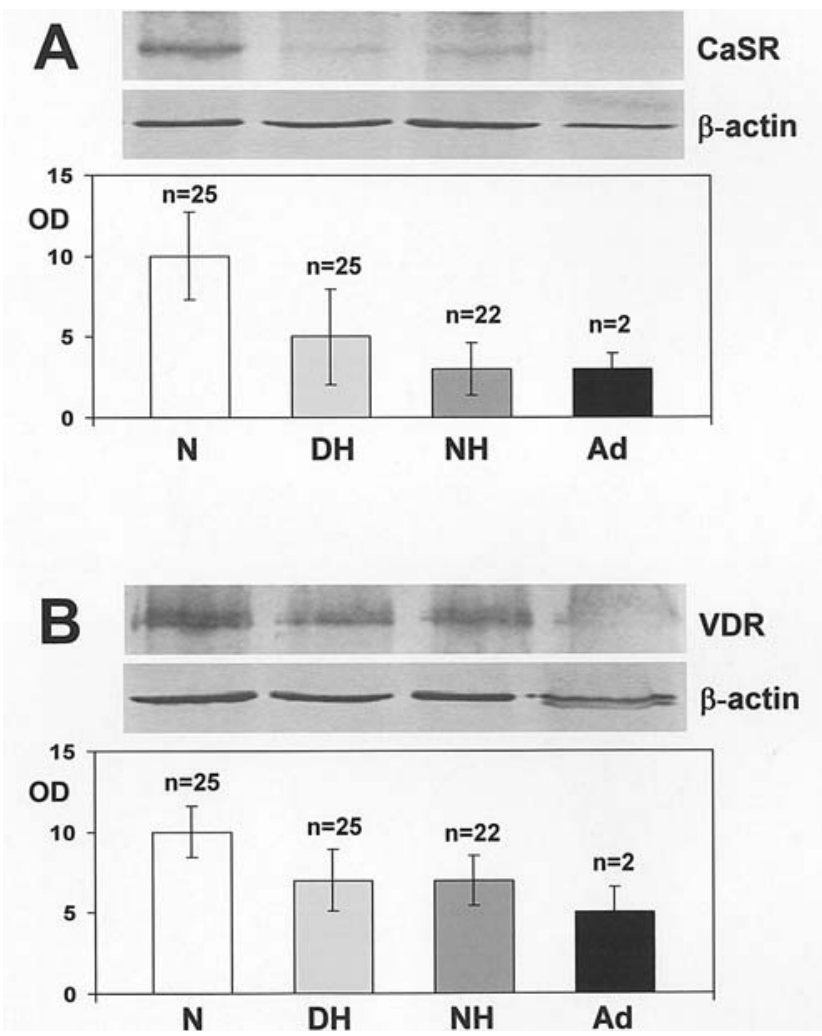

Figure 2. Expression of the CaSR (panel A) and VDR (panel B) in the parathyroid gland biopsies assessed by Western blot analysis. Representative samples of each histological type are shown. The histograms represent the results (mean $\pm \mathrm{SD}$ ) of semiquantitative densitometric CaSR and VDR expression analysis (adjusted to $\mathrm{B}$-actin expression). N, normal; DH, diffused hyperplasia; $\mathrm{NH}$, nodular hyperplasia; Ad, adenoma; $\mathrm{OD}$, relative optical density.

In 17 of 19 patients, the origin of kidney insufficiency was chronic glomerulonephritis. In 2 individuals, the kidney insufficiency developed as a complication of diabetes. The study was performed with the formal approval of the Local Ethics Committee, and all patients gave their informed consent to participate.

Tissue preparation. The surgical procedure concerned subtotal parathyroidectomy. Immediately after the excision of parathyroid glands and prior to fixation, small fragments of parathyroid tissue (approximately $20-50 \mathrm{~mm}^{3}$ ) were homogenized in lysing buffer (10 mM Tris, $150 \mathrm{mM} \mathrm{NaCl}$, $1 \%$ Triton $\mathrm{X}-100$, and $0.1 \%$ SDS) with a cocktail of protease inhibitors (Roche Diagnostics, Basel, Switzerland) and stored at $-70^{\circ} \mathrm{C}$ until use in Western blot analysis. The remaining material was fixed in buffered formalin and underwent routine histological preparation. The hematoxylin-eosinstained sections were subjected to microscopic examination, and their morphological features were determined (14). As a control, normal parathyroid glands obtained during cadaver donor multi-organ harvesting were used.

Western blotting. The tissue homogenates, $10 \mu \mathrm{g}$ of total protein per line, were run in $12 \%$ polyacrylamide vertical gel using standard SDS-PAGE protocol (15). The separated proteins were transferred onto polyvinylidene difluoride (PVDF) membranes using a semi-dry blotting method 


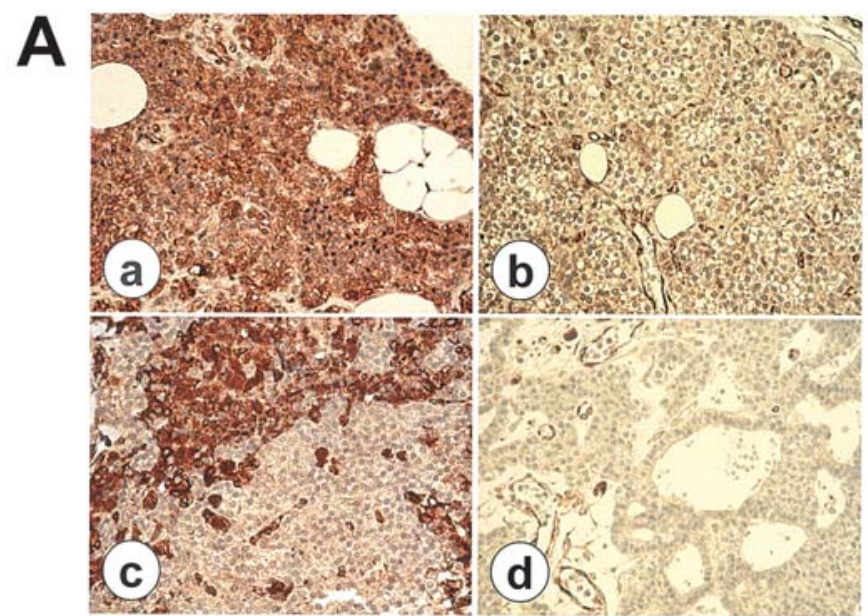

B

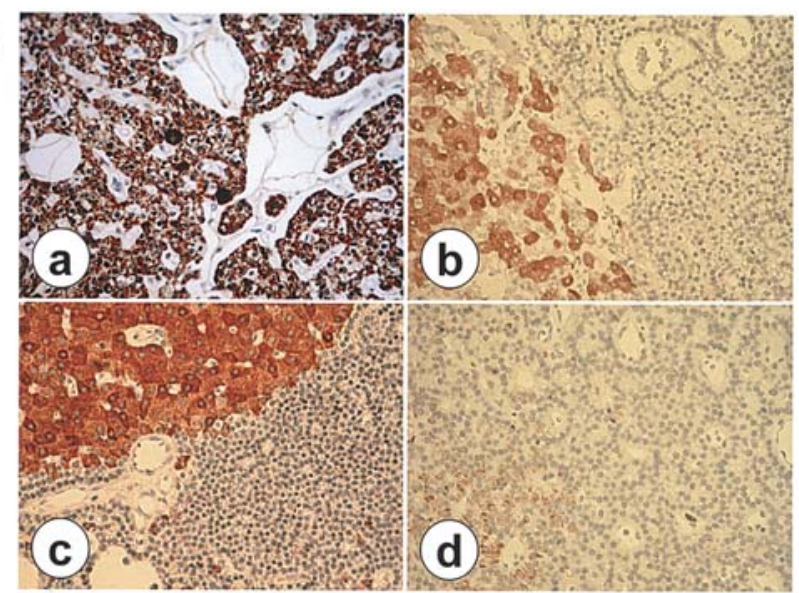

Figure 3. The expression of the CaSR (panel A) and VDR (panel B) in the parathyroid gland specimens immunostained with anti-CaSR or anti-VDR antibody, respectively. A representative sample of each histological type is shown (original magnification x200): a, normal; b, diffused hyperplasia; c, nodular hyperplasia; d, adenoma.

(Bio-Rad, Hercules, CA). PVDF membranes were then incubated with rabbit anti-CaSR or rat anti-VDR monoclonal antibodies (both from Chemicon, Temecula, CA). The specific proteins were detected with an alkaline phosphatase system (Bio-Rad), and the membranes were dried and their digital pictures were taken. The amount of specific reaction products was analyzed using Grab-IT 2.0 software (UVP, Cambridge, UK). For the semi-quantitative analysis of CaSRand VDR-specific products, the protein amount loaded for electrophoresis was verified on the basis of $\beta$-actin expression, and detected with the respective mouse monoclonal antibody clone AC-15 (Sigma-Aldrich, St. Louis, MO).

Immunohistochemisty. Formalin-fixed paraffin-embedded sections were deparaffinized and re-watered, then washed with PBS followed by incubation with anti-CaSR rabbit or anti-VDR rat monoclonal antibodies (both from Chemicon). Afterwards, the reaction was revealed with the EnVision horseradish peroxidase system and diaminobenzidine (Dako, Glostrup, Denmark). The immunostained sections were assessed using a Nikon Eclipse E800 microscope equipped with a Nikon 995 Coolpix 3.38 MPx digital camera (Nikon, Japan). Digital pictures (original magnification, x100) were analyzed using Grab-IT 2.0 software (UVP) as previously described (16). The final intensity of the immunostaining reaction was calculated as a mean result of individual measurements in a 25-point matrix overlaying the picture.

\section{Results}

Histology of parathyroid glands. Among 64 parathyroid glands harvested during surgery from T-HPTH patients, 58 parathyroids revealed the benign hyperplasia morphology. They were further classified as diffused $(\mathrm{DH} ; \mathrm{n}=25)$, nodular $(\mathrm{NH} ; \mathrm{n}=22)$ or unclassified/mixed $(\mathrm{UH} ; \mathrm{n}=11)$ hyperplasia. In one patient, two glands were identified as adenoma (Ad). The remaining 4 of 64 studied glands did not reveal any signs of pathology (Fig. 1).

Interestingly, in 12 of 19 T-HPTH patients in this study, we found both types of hyperplasia. Moreover, in 5 of them we observed the coexistence of $\mathrm{DH}$ and $\mathrm{NH}$ features in the same gland. Furthermore, 3 of 19 patients had only DH-classified parathyroids, whereas 3 other individuals possessed only NH glands. No significant correlations between the histology of glands and plasma levels of calcium and/or PTH were observed. The mean calcium plasma level in the T-HPTH group was $3.0 \pm 0.25 \mathrm{mM}$, with a mean PTH plasma level of $315 \pm 338 \mathrm{pg} / \mathrm{ml}$ and a median value of $187 \mathrm{pg} / \mathrm{ml}$.

The cadaver donor-derived parathyroid glands $(n=21)$ displayed normal morphology, with the exception of one parathyroid characterized as mild UH.

Western blot analysis. Western blot analysis of the CaSR protein revealed a relatively low receptor amount in samples of homogenized parathyroid glands from T-HPTH patients. In contrast, samples of parathyroid tissue from control glands of cadaver donors, as well as 4 normal glands from T-HPTH patients, displayed a strong CaSR-specific signal (Fig. 2A). No significant correlation between the type of hyperplasia (including two adenoma-type glands) and CaSR expression level in the T-HPTH group was observed.

The analysis of VDR protein expression in parathyroid homogenates revealed a similar pattern to that obtained in the case of CaSR (Fig. 2B). Again, no correlation between the hyperplasia type and receptor protein expression level in the patient group was found.

Immunostaining. The immunostaining of T-HPTH patientderived hyperplastic parathyroid tissue revealed its pronounced diversity in the expression of both CaSR (Fig. 3A) and VDR (Fig. 3B). Interestingly, in the same section, small regions with a very high expression of CaSR or VDR (assessed as grade 3 or 4) were surrounded by large areas of less intensively stained hyperplastic tissue (estimated as 0 or 1). This pattern resulted in significantly lower overall receptor expression levels, as calculated using a 25-point measurement matrix. In contrast, the control parathyroid glands displayed univocally positive and relatively uniform staining patterns for both CaSR and VDR (Fig. 3A and B, respectively).

Contrary to the results of Western blot analysis, the semiquantitative analysis of immunostained sections showed a significantly different mean expression level of both CaSR and VDR, depending on the histological structure of 

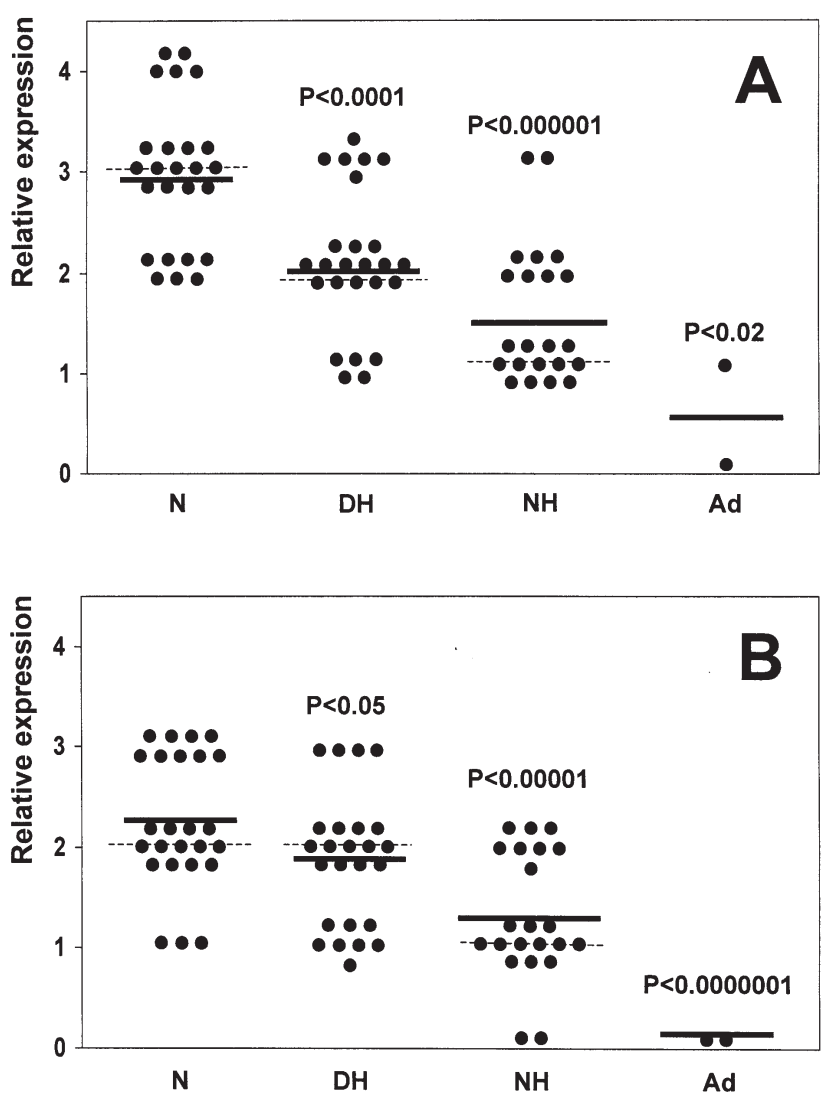

Figure 4. Semiquantitative analysis of the CaSR (panel A) and VDR (panel B) expression in the immunostained parathyroid gland specimens. Each parathyroid gland is shown as one dot on the graph. The horizontal lines correspond to the mean expression value, and dashed lines represent the median for the group. The number of glands in each group is shown as $\mathrm{n}=$ value. $\mathrm{N}$, normal; $\mathrm{DH}$, diffused hyperplasia; $\mathrm{NH}$, nodular hyperplasia; Ad, adenoma. Statistical analysis was performed using the Mann-Whitney U-test.

parathyroids (Fig. 4A and B). Moreover, we found a statistically significant difference in CaSR and VDR expression between both types of hyperplasia ( $p=0.009$ and $p=0.005$, respectively). Although the difference in CaSR expression between $\mathrm{NH}$ and Ad-type parathyroids was statistically non-significant, this presumably was due to the small number of the latter histological type. Thus, the CaSR and VDR expression levels gradually decreased (in order from highest to lowest): $\mathrm{N}>$ $\mathrm{DH}>\mathrm{NH}>$ Ad.

\section{Discussion}

Activation of CaSR and VDR molecules results in the suppression of PTH production and/or secretion $(1,17)$. Therefore, the impaired expression of one or both receptors may lead to PTH overproduction and the development of clinical symptoms of HPTH. However, the reason behind the decrease in CaSR and/or VDR expression in parathyroid glands remains unclear.

It is possible that the decrease in receptors is a causative event, resulting in the constant stimulation of parathyroid cells, their proliferation and PTH hypersecretion, as observed in P-HPTH. On the other hand, one cannot exclude that impaired expression of CaSR and VDR could be a secondary effect of metabolic abnormalities associated, e.g. with kidney insufficiency, as found in S-HPTH, or other yet unidentified molecular changes associated with T-HPTH. Interestingly, the transgenic mice experiments with parathyroidtargeted overexpression of cyclin D1 provided evidence that impaired CaSR expression could be a consequence of parathyroid cell proliferation (18). Other mechanisms, including calpain-mediated CaSR degradation might be considered (19). Furthermore, impaired expression of VDR could be induced by PTH, a known down-regulator of the receptor for vitamin D (20). Thus, both points of view have their merits. It is also plausible that both mechanisms may be simultaneously involved in the development of T-HPTH.

This hypothesis could be supported by the observed high heterogeneity of the parathyroid cell population, at least in context of the expression of both receptors found in the T-HPTH glands. In the same specimen, CaSR- or VDRnegative cells as well as cells with high expression of both receptors could be detected. The reason for such differences within a single gland is unknown. It might suggest the polyclonal origin of autonomous regions in T-HPTH parathyroids or indicate that some compensatory mechanisms of receptor expression regulation take place. Such differences could explain the observed discrepancy between the results of immunohistochemistry and Western blot analysis. This could be due to the random derivation of small parathyroid tissue pieces used for preparing Western blot homogenates. Due to the high risk of misreading receptor expression, such biopsies should not be used for quantitative evaluation, instead immunostained serial histological specimens should be used.

Regardless of the reduced expression of both CaSR and VDR, it is difficult to clearly predict which of the receptors is of greater importance for the development of T-HPTH. Surprisingly, the experiments with calcimimetic agents (activating ligands for CaSR, e.g. cinacalcet hydrochloride) showed that despite the considerable decrease of receptor protein expression, signalling through the CaSR is substantially preserved and results in a significant reduction of the plasma PTH level (21). These observations, together with data indicating the cross-regulation of CaSR and VDR expression, suggest the superior role of VDR over CaSR. Moreover, Giannini and co-authors proposed that the major mechanism responsible for the development of HPTH could be the polymorphism of the VDR gene (22). However, further studies on parathyroid glands are required to verify this thesis and to elucidate the observed expression patterns of CaSR and VDR molecules in T-HPTH in detail.

\section{Acknowledgements}

The study was supported by grant no. 6 P05C 03120 from the State Committee for Scientific Research and individual research grants from the Warsaw Medical University.

\section{References}

1. Chen R and Goodman W: Role of the calcium-sensing receptor in parathyroid gland physiology. Am J Physiol Renal Physiol 286: 1005-1011, 2004.

2. Ruat $\mathrm{M}$ and Petrel $\mathrm{C}$ : New insights into the pharmacology of the extracellular calcium sensing receptor. Med Sci 20: 980-985, 2004. 
3. Wada M, Furuya Y, Sakiyama J, Kobayashi N, Miyata S, Ishii H and Nagano N: The calcimimetic compound NPS R-568 suppresses parathyroid cell proliferation in rats with renal insufficiency. Control of parathyroid cell growth via a calcium receptor. J Clin Invest 100: 2977-2983, 1997.

4. Thakker R: Diseases associated with the extracellular calciumsensing receptor. Cell Calcium 35: 275-282, 2004.

5. Pramanik R, Asplin JR, Lindeman C, Favus MJ, Bai S and Coe FL: Lipopolysaccharide negatively modulates vitamin D action by down-regulating expression of vitamin D-induced VDR in human monocytic THP-1 cells. Cell Immunol 232: 137-143, 2004.

6. Sunn KL, Cock TA, Crofts LA, Eisman JA and Gardinger EM: Novel N-terminal variant of human VDR. Mol Endocrinol 15: 1599-1609, 2001

7. Llach F: Secondary hyperparathyroidism in renal failure: the trade-off hypothesis revisited. Am J Kidney Dis 25: 663-679, 1995.

8. Koch Nogueira PC, David L and Cochat P: Evolution of secondary hyperparathyroidism after renal transplantation. Pediatr Nephrol 14: 342-346, 2000

9. Younes NA, Shafagoy Y, Khatib S and Ababneh M: Laboratory screening for hyperparathyroidism. Clin Chim Acta 353: 1-12, 2005.

10. Kwak JO, Kwak J, Kim HW, Oh KJ, Kim YT, Yung SM and Cha SH: The extracellular calcium sensing receptor is expressed in mouse mesangial cells and modulates cell proliferation. Exp Mol Med 37: 457-465, 2005.

11. Rodriguez M, Nemeth E and Martin D: The calcium-sensing receptor: a key factor in pathogenesis of secondary hyperparathyroidism. Am J Physiol Renal Physiol 288: 253-264, 2005.

12. Brown EM: Physiology and pathophysiology of the extracellular calcium-sensing receptor. Am J Med 106: 238-253, 1999.

13. Sudhaker Rao D, Han ZH, Phillips ER, Palnitkar S and Parfitt AM: Reduced vitamin D receptor expression in parathyroid adenomas: implications for pathogenesis. Clin Endocrinol 53: $373-381,2000$
14. Grimelius L, Akerstrom G, Bondeson L, Juhlin C, Johansson H, Ljunghall $\mathrm{S}$ and Rastad J: The role of the pathologist in diagnosis and surgical decision making in hyperparathyroidism. World $\mathbf{J}$ Surg 15: 698-705, 1991.

15. Lazarczyk M, Dziunycz P, Niderla J, et al: Influence of pentoxifylline on natural cytotoxicity and expression of granzymes and PI-9, a specific granzyme B inhibitor. Int J Mol Med 17: 135-140, 2006

16. Chudzinski W, Niderla J, Lasiecka Z, et al: P-glycoprotein expression influences the result of $99 \mathrm{mTc}-\mathrm{MIBI}$ scintigraphy in tertiary hyperparathyroidism. Int J Mol Med 16: 215-220, 2005.

17. Dusso AS, Thadhani R and Slatopolsky Y: Vitamin D receptor and analogs. Semin Nephrol 24: 10-16, 2004

18. Yano S, Sugimoto T, Tsukamoto T, et al: Association of decreased calcium-sensing receptor expression with proliferation of parathyroid cells in secondary hyperparathyroidism. Kidney Int 58: 1980-1986, 2000.

19. Kifor O, Kifor I, Moore FD, Butters RR and Brown EM: M-calpain colocalizes with the calcium-sensing receptor $(\mathrm{CaR})$ in caveolae in parathyroid cells and participates in degradation of the CaR. J Biol Chem 33: 31167-31176, 2003.

20. Healy KD, Vanhooke JL, Prahl JM and DeLuca HF: Parathyroid hormone decreases renal vitamin $\mathrm{D}$ receptor expression in vivo. Proc Natl Acad Sci USA 102: 4724-4728, 2005.

21. Mizobuch M, Hatamura I, Ogata H, et al: Calcimimetic compound upregulates decreased calcium-sensing receptor expression level in parathyroid glands of rats with chronic renal insufficiency. $\mathrm{J}$ Am Soc Nephrol 15: 2579-2587, 2004.

22. Giannini S, D'Angelo A, Nobile M, et al: The effects of vitamin $\mathrm{D}$ receptor polymorphism on secondary hyperparathyroidism and bone density after renal transplantation. J Bone Miner Res 17: 1768-1773, 2002. 